\title{
HOMOFOBIA: APRECIACIONES DESDE TRES PERSPECTIVAS PSICOLÓGICAS
}

\section{HOMOPHOBIA: APPRECIATIONS FROM THREE PSYCHOLOGICAL PERSPECTIVES}

\author{
José Alonso Andrade-Salazar ${ }^{1}$, Ana María Borja-Acosta², Jennifer Andrea Soto-Cardona ${ }^{3}$, \\ Sandra Patricia Camelo-Osorio ${ }^{4}$
}

\section{RESUMEN}

La presente investigación busca aproximarse a las causas psicosociales de la homofobia a través de una investigación cualitativa, con base al paradigma comprensivo-hermenéutico abordada desde los principios epistemológicos de tres escuelas psicológicas: psicoanálisis, humanismo y conductual. En la región latinoamericana, la homofobia es un fenómeno concebido aún como tabú, diferentes víctimas de este tipo de discriminación han recibido por parte de los victimarios agresiones de diversa índole. Estos aspectos pueden explicarse por efectos del reforzamiento de conductas por parte de la familia, grupo de pares y diversas representaciones sociales en el plano biopsicosocial. En síntesis, la homofobia es una problemática real que a la fecha perturba la salud mental y física de personas homosexuales, al provenir de un sistema en donde la homogeneidad y la "normalidad" es lo prioritario y fundamental para encajar dentro de una sociedad.

Palabras clave: Homofobia; homosexualidad; discriminación; psicología; Latinoamérica

\section{ABSTRACT}

This research seeks to explore the psychosocial causes of homophobia using qualitative research methods to interpret the paradigm of hermeneutics through the epistemology principles of focus groups, observation, and interviews. In the Latin American region, homophobia is still conceived as an acceptable practice, victims of this type of discrimination have been victims of various heinous acts perpetrated by homophobic members of society. These actions are ultimately the consequences of social reinforcement of these negative behaviors through family, school peers, co-workers, and other groups encountered within their social plane. In a society where "normality" and homogeneity are a priority, homophobiais a threat to the mental and physical health of homosexual community members within a system that desires to force normality on them in order to be accepted members of society.

Keywords: Homophobia; homosexuality; discrimination; psychology; Latin America

Tipología: Artículo de investigación científica y tecnológica

Fecha de recepción: 18/06/2015

Fecha de aceptación: 23/11/2015

Como citar este artículo: Andrade-Salazar, J.A., Borja-Acosta, A.M., Soto-Cardona J.A. \& Camelo-Osorio, S.P. (2016). Homofobia: Apreciaciones desde tres perspectivas psicológicas. Jangwa Pana, 15 (1), 13 - 27

1. Psicólogo. Docente investigador y coordinador de investigaciones. Investigador del grupo interdisciplinario para el desarrollo y la acción dialógica (GIDPAD) y del grupo Estudios clínicos y sociales en psicología de la Facultad de psicología. Universidad de San Buenaventura Medellín extensión Armenia. Colombia Correo electrónico: jose.andrade@usbmed.edu.co; 911psicologia@gmail.com

2. Ana María Borja Acosta. Estudiante de X semestre de la Facultad de Psicología de la Universidad de San Buenaventura Medellín extensión Ibagué. Colombia. Correo electrónico: annye-92@hotmail.com.

3. Jennifer Andrea Soto Cardona. Estudiante de X semestre de la Facultad de Psicología de la Universidad de San Buenaventura Medellín extensión Ibagué. Colombia. Correo electrónico: gitik_9322@hotmail.com

4. Sandra Patricia Camelo Osorio. Estudiante de X semestre de la Facultad de Psicología de la Universidad de San Buenaventura Medellín extensión Ibagué. Colombia. Correo electrónico: sandracamelo23@gmail.com 


\section{INTRODUCCIÓN}

$\mathrm{E}$ n una sociedad que exige la masculinidad y la femineidad como aspecto relevante en la identidad de hombres y mujeres, la emergencia de tendencias alusivas a la homosexualidad, es decir, a la atracción por personas del mismo género, puede resultar contradictoria y en algunos casos amenazante (Rivas, 2012). A menudo las elecciones afectivas de personas homosexuales genera un rechazo visible a través de manifestaciones violentas tales como señalamientos, exclusión, agresiones verbales, violaciones e incluso asesinatos (Espejo, 2012). En el período concebido entre los siglos XVI y XVII, algunos países europeos atravesaron tiempos de abominación contra la penetración anal entre hombres homosexuales, práctica connotada como "sodomita" y relación sexual aberrante (Badinter, 1993), esta tendencia se mantuvo y aún existen rezagos de esta prohibición en la cultura; pese a ello el mundo occidental paulatinamente experimentó una crisis de los roles tradicionales que aumentó la tendencia a buscar nuevas formas de interacción afectiva, al tiempo que actitudes homofóbicas en hombres heterosexuales; consecuencia de ello fue el acrecentamiento del individualismo heterosexual, como respuesta negativa ante la expresión personal de la identidad homosexual, a fin de defender la autonomía de la familia tradicional y rechazar las sexualidades diferentes (Christopher \& Sprecher, 2000). En este tópico la discriminación con base en la diferencia de la orientación sexual, se constituyó en una constante en grupos y personas arraigadas al autoritarismo y la sumisión.

La homosexualidad puede ser definida como una tendencia o elección de prácticas e intenciones afectivas y eróticas dirigidas hacia personas del mismo género, vinculada a elementos instintivos, afectivos, aprendizajes y elecciones, así como también a aspectos culturales, ambientales, genéticos y/o educativos (Espejo, 2009). La homosexualidad suscita asombro y rechazo, en consecuencia, la discriminación busca anular en las "minorías sexuales" el derecho a la libre expresión, lo cual se extiende hasta acciones de desaprobación de derechos como el matrimonio, conformación de familias, adopción, y vinculación de las mismas a entidades de salud, entre otras (Rodríguez, 2012); dichas acciones e complementan con expresiones de violencia física, relacional y psicológica hacia personas homosexuales (Chamberland \& Lebreton, 2012). Existe en los homofóbicos una relación directamente proporcional entre la edad de los sujetos y la magnitud de las actitudes negativas dirigidas hacia los homosexuales (a mayor edad mayor discriminación); en personas heterosexuales esto se invierte ya que la discriminación es inversamente proporcional a la edad y nivel de escolaridad, ydirectamente proporcional a la cantidad de prejuicios en la persona homosexual (Gomez, 2007).

Para la psicología, la sexualidad es aquello que permite la existencia de lo sexual y del sexo, lo que incluye también las elecciones de los sujetos respecto a las diversas formas de ejercicio de su sexualidad (Ardila, 1998). En este rango de definiciones el concepto de orientación sexual es crucial, así para la APA (2008) la orientación sexual son todas aquellas acciones permanentes respecto al hecho de disfrutar y elegir formas de vivir lo sexual, lo cual referencia no solo un sentimiento constante hacia otra persona, sino una identidad sexual que define ciertos comportamientos de acuerdo a personas, grupos y espacios. Entre las orientaciones se encuentran: heterosexualidad, homosexualidad, bisexual y transexual (APA, 2008). Lacarde (1996) considera que la no aceptación violenta de formas no-tradicionales de vivir lo sexual y lo afectivo conlleva al sexismo, el cual es identificable a través del machismo, el androcentrismo, la misoginia y la homofobia, que tienen en común un trasfondo patriarcal y discriminatorio de base. En este sentido el género no es un producto terminado de la biología, sino un proceso en el que las personas pueden ir eligiendo el sentido sexual de sus experiencias, a partir de la interacción con otras personas (Ardila, 1998); sin embargo, la remarcación social de las diferencias entre masculinidad y feminidad agrandan 
la brecha de la aceptación de la homosexualidad, siendo ambas justificantes de la mayoría de actos de homofobia (Lamas, 2000).

La Organización de las Naciones Unidas para la Educación, la Ciencia y la Cultura (UNESCO, 2015) el fenómeno de la homofobia es tan creciente que incluso en el ámbito de las instituciones educativas se presenta el bullying homosexual, el cual dirige su atención a personas homosexuales, acosándolas y causando daño físico, psicológico y moral a menudo irreversible. Es importante señalar que algunas instituciones se oponen a la homofobia promoviendo los derechos de las personas homosexuales como respuesta a las amenazas de los homofóbicos, ejemplo de ello son las campañas de prevención dignificación y educación sexual promovidas por el Instituto Nacional contra la Discriminación, la Xenofobia y el Racismo (INADI, 2015), que propenden por desmitificar las formas de elección sexual diferente. En Latinoamérica suele presenciarse con frecuencia acciones agresivas contra personas homosexuales, ambisexuales, transgénero e intersexuales (OPS, 2006). De acuerdo con Carrillo (2006) la ambisexualidad y bisexualidad son distintas, ya que mientras la ambisexualidad significa la atracción sexual referida a individuos de ambos géneros, la segunda refiere las características masculinas y femeninas presentes en todas las personas, con mayor preponderancia de hormonas masculinas en hombres, y femeninas en mujeres o casos inversos. De acuerdo a la Asociación Internacional de Lesbianas, Trans, Gays, Bisexuales (ILGA), Colombia y Brasil son los países más afectados por el fenómeno de la homofobia, ya que entre el 2008 y el 2011 se produjeron en ambos países las mayores tasas de eventos de agresión y discriminación (ILGA, 2013), al tiempo que Perú presenta la mayor variedad de expresiones de homofobia entre los 138 países del mundo. El fenómeno de la homofobia es tan nocivo que en Latinoamérica al menos el $40 \%$ de personas homosexuales y el $65 \%$ de los transexuales fue víctima violencia homofóbica en el ámbito escolar (UNESCO 2015a).
Según el informe realizado por Colombia Diversa en el periodo 2006-2007, 67 personas de la comunidad de Lesbianas, gais, bisexuales y personas transgénero (LGBT) fueron asesinadas por motivo de la intolerancia. Entre el 2013 y 2014 se registraron 164 homicidios en los 25 departamentos del país, especialmente en Antioquia, Valle del Cauca y Bogotá (Colombia diversa, 2013), de ellos 30 crímenes habrían estado motivados a razón del prejuicio respecto a la orientación sexual o identidad de género de las víctimas. "Sin embargo, dada la precariedad de la información disponible para la mayoría de casos, el número de homicidios por prejuicio podría ser más alto" (p. 2). En Nicaragua, la homofobia se expresa con casos de violación a 2 de cada 10 personas pertenecientes a la comunidad LGBTI (lesbianas, gais, bisexuales, transgénero e intersexuales) (García, Quiroga, Morgan, Paller \& Marrero, 2011). Asimismo, en México las expresiones homófobas son elevadas; la Encuesta Nacional de Cultura Política y Prácticas Ciudadanas (Citada en Mondragón, 2009) mostró que el 66\% de los mexicanos no vivirían en el mismo hogar con un homosexual y $71 \%$ de los jóvenes no apoyarían los derechos de los homosexuales. En las encuestas del Consejo Nacional para Prevenir la discriminación (CONAPRED) aplicadas a homosexuales, el 95\% indicó sentirse discriminado, cuatro de cada diez excluidos, más del $50 \%$ rechazados y "el 60\% afirmaron que consideran que su principal enemigo es la sociedad" (Mondragón, 2009, p. 132).

Desde una perspectiva legal se encuentra que muchos derechos de los homosexuales son violados y a menudo las leyes no se aplican en temáticas referentes a discriminación y exclusión (Ballero, 2008), aspecto visible en que sólo algunos países han avalado leyes que legitiman uniones civiles y el matrimonio homosexual (García, et al., 2011).

Los países de América Latina y el Caribe que son precursores en despenalizar las relaciones sexual/afectivas entre personas del mismo sexo fueron Brasil (1831), México (1872) y 
Argentina (1887). Después seguiría Perú (en 1924), y poco, más tarde Uruguay (1934). 47 años después, en 1971, se les uniría Costa Rica, en 1979 Cuba y en 1981 Colombia. En la década de los ' 90 otros dos países, Ecuador (1997) y Chile (1998) despenalizaron la homosexualidad. Los últimos países en hacerlo fueron Nicaragua y Panamá (2008). (García, et al, 2011, p. 7)

En todo el territorio de América Latina, la homosexualidad se encuentra despenalizada, e inclusive en algunos países de la zona han adoptado legislaciones para sancionar las discriminaciones y agresiones sufridas por causa de intolerancia frente a la diversidad de orientación sexual y violencia de género (Paulón, 2010). En este artículo la homofobia busca ser comprendida de forma relacional desde un paradigma comprensivo-hermenéutico, con base en aportes de los enfoques cognitivo-conductual, psicoanalítico y humanista, lo cual orienta este trabajo desde la disciplina psicológica. El comportamiento fóbico solo es una parte de las posturas explicativas, ya que la conducta discriminante va desde tensiones de género, imaginarios sociales sobre los roles y la reproducción de la especie, prejuicios, temores y la estigmatización de las personas homosexuales.

\section{MATERIALES Y MÉTODOS}

\section{Diseño}

Esta es una investigación cualitativa descriptiva de corte interpretativa, cuyo objetivo principal es realizar una revisión de la forma en que las tres principales posturas o escuelas psicológicas ven la homofobia. Para ello se recurrió a publicaciones ubicadas en las bases de datos Redalic, EBSCO, Latindex, APA PsycNET, Google Académico y SciELO, además de libros, informes y periódicos. Se revisaron 50 artículos en español (5 en inglés) publicados en el periodo 2002-2014. Existen también referencias de auto- res clásicos que anteceden a dicho periodo y que deben incluirse dada la información requerida en el trabajo investigativo. Las palabras clave que guiaron la búsqueda fueron: LGTB, homosexualidad, discriminación, enfoques psicológicos. La selección de la información relevante para el estudio se realizó a través de rejillas en las que se sistematizó la información de acuerdo a su aporte y nivel de importancia.

\section{RESULTADOS}

Después de la revisión de los artículos científicos, libros y noticias se encontró que los estudios se centran especialmente en la necesidad de restitución de los derechos de las personas homosexuales por parte de organizaciones y las mismas comunidades LGBT, cabe anotar que en todos estos trabajos se denuncian los abusos de poder y acciones de violencia homofóbica en contra de la integridad de las personas homosexuales (Herek \& Berrill, 1992; Paulón, 2010; Izaguirre, 2008; Velásquez, 2008; Mondragón, 2009; Bustamante, 2009; CEPRESI, 2009; García, et al., 2011; Colombia Diversa, 2013; INADI, 2015). Algunas investigaciones se centran en la identidad de femenina/masculina para comprender la forma como se interiorizan los roles y la elección de género, así como también explicaciones acerca de las acciones de discriminación (Badinter, 1993; Christopher \& Sprecher, 2000; Mott, 2005; ILGA, 2013). Las explicaciones que los trabajos de investigación dan a la homosexualidad suelen ser multi-fenoménicos y reiteran la condición de elección de las personas sobre su condición sexual (Valores, 2002; Penas, 2008; Carrillo, 2006; OPS, 2006; Espinoza, 2011; García, et al, (2011). De todos los enfoques psicológicos que muestran interés por la homosexualidad, es el enfoque psicoanalítico el que tiene más trabajos publicados. Para el psicoanálisis la persona puede tener tendencias inconscientes que lo impulsan a la homosexualidad, asimismo, sobre-identificación con la madre (gais) o el padre (lesbianas), un Edipo negativo, o 
experiencias tempranas de homosexualidad pueden propiciar esta elección

El enfoque cognitivo-conductual también ha realizado estudios sobre la homosexualidad pero se centran en el aprendizaje de este tipo de conductas, reduciendo el fenómeno a la influencia del ambiente, y posteriormente a la interinfluencia de otros (Almansa, 2011; Rivas, 2012; Navas, 1981). Para el humanismo la elección es una aptitud vital inalienable ligada a la autenticidad, sin embargo las personas que ocultan su homosexualidad suelen sufrir una crisis de identidad que los lleva a vivir de formas no-auténticas, lo cual causa dolor y en ocasiones problemas en el sentido existencial. En general los tres enfoques presentan un hilo conductor que los asocia: la idea de que las conductas homófobas son el producto de problemas de la identidad del sujeto, sea cual sea su origen emergencia o elección, al tiempo que toda elección homosexual no surge dela nada, ella es el resultado de un proceso de concienciación de la forma como el sujeto proyecta sus vínculos afectivos y su desarrollo sexual (Andrade, Quintero \& Téllez, 2012). Existen también investigaciones acerca de la influencia de la homofobia en la salud (Soberon \& Feinzhol, 2007), las implicaciones familiares de la homofobia y de la homosexualidad en las interacciones familiares (Rodríguez-Castro, Lameiras-Fernández, Carrera-Fernández \& Vallejo-Medina, 2013) y Bullying homófobo (UNESCO, 2015).

\section{Posición del psicoanálisis respecto a la homofobia}

Las relaciones homosexuales aún no son aceptadas por algunos sectores sociales, específicamente en la cultura occidental, donde muchos individuos suelen ser víctimas de discriminación, castigos y rechazos. Estas conductas ocasionan desajustes en el funcionamiento del yo en el principio de realidad, el cual depende de condiciones sociales, percepciones de conjunto sobre la sexualidad, mecanismos defensivos, identificaciones, introyecciones y la influencia del súper yo para dar sentido a las vivencias (Izaguirre, 2008). Según Sigmund Freud (1923) el súper yo es la estructura psíquica encargada de funciones tales como la censura, participa en la represión, la conciencia moral y la formación de ideales. En la estructuración y formación del súper yo de un sujeto homofóbico es importante resaltar la influencia de los factores ambientales y hereditarios, incluyendo el temperamento, la moralidad y la ética, entre otros. El súper yo se estructura con la resolución del complejo de Edipo, al convertirse el sujeto en heredero de los comportamientos normativos de la economía psíquica influida por el "ideal del yo", concebido éste como una subestructura preexistente al súper yo consolidado. El ideal del yo se ajusta a las normas sociales establecidas por los distintos medios de interacción social en la infancia: el colegio, los pares, la religión y la familia (Gaione, 2009), mientras el Yo ideal puede distar de éste porque referencia las intenciones reales de las personas, que en la homofobia pueden indicar en algunos casos un fuerte deseo de integración de la homosexualidad a sus vidas.

El súper yo individual y toda la estructura de respuesta psicológica de una persona homofóbica se veinfluenciada por las normas y convenciones sociales (Andrade et al., 2012) pues a partir del sistema de creenciasse forman inicialmente los prejuicios y culpas que instauran la homosexualidad en el marco de la transgresión. Las pautas de crianza rígidas pueden propiciar el desarrollo de un yo inmaduro cuyo súper yo sea controlador, dominante y culposo. El súper yo en el sujeto homofóbico opera a través del sentimiento de culpa inconsciente que lo torna propenso asufrir de notables compulsiones y prohibiciones, así la persona para evitar o tramitar precariamente estos afectos disfuncionales suele actuar a partir de su prohibición, es decir, en función de la exclusión de las sexualidades diferentes. En la cultura latinoamericana, la comunidad homosexual aún no es bien vista por la mayoría debido a las regulaciones morales que imperan en los sistemas de crianza, en este sentido los homófobos se encuen- 
transometidos a peticiones del súper yo que impiden aceptar la homosexualidad como parte de las elecciones personales, como respuesta se crea una aversión hacia estas "minorías sexuales".

El inconsciente freudiano, se compone en parte de representaciones y afectos reprimidos a lo largo de la historia del sujeto, cuyas experiencias pulsionales se memorizan y se cargan energéticamente (Freud, 1978), algunas de ellas incluyen experiencias homofóbicas que luego se convierten en sentimientos y acciones apuntaladas por los grupos con los cuales se interactúa. En las representaciones priman aspectos idiosincrásicos, afectos, culturales, experiencias y reproducciones de modelos y pensamientos acerca de una realidad experimentada colectivamente (Moscovici, 1999; Araya, 2002), motivo por el cual resultan determinantes para comprender la forma como la homofobia se extiende en personas y colectivos hasta legitimarse en estos.

La novela familiar sería entonces, la sustitución que hacen los individuos neuróticos de su familia real por una familia imaginada que le satisficiera las necesidades o le compensara frustraciones estableciendo esto en los grupos a través de la identidad grupal, la cohesión y el sentido de pertenencia (Andrade, 2011). Si bien la homofobia se estructura como conducta desde las posibilidades de identificación del yo, no se puede reducir el fenómeno a la crianza o la influencia social, ya que existen otros factores socioculturales e incluso políticos que la fomentan. Ortega (2011) señala que la familia ideal no existe, y "menos aún la familia natural puesto que tanto la maternidad como la paternidad son del orden del significante. Si la familia es sólida es justamente porque está engendrada por un símbolo que es a su vez su vehículo" (Ortega, 2011, p.5). Para el psicoanálisis la familia debe hacer al individuo un sujeto del deseo y ofrecer un lugar simbólico de inclusión del otro, junto a lazos de identificación parental positivos y el desarrollo de la independencia emocional. Ergo, la familia es fuente de "humanización del deseo", exaltación de las pulsiones que admite al individuo instalarse en un lazo social de vida, cuando sus capacidades de acción, creación y de amor pueden satisfacerse, así cuando la familia no fomenta la inclusión y la tolerancia, todo aquello que resulta diferente se convierte en amenaza para los sujetos, situación visible en personas homofóbicas.

\section{Posición del modelo cognitivo-conductual respecto a la homofobia}

De acuerdo con Ellis, los estados emocionales no son producto de los acontecimientos en sí, sino de la significación que se les atribuye; de este modo, por ejemplo, si (B) es la significación de (A), es desde (B) que se originan los estados emocionales (C). Por ende al modificar los esquemas mentales (D) se producirán estados emocionales que mantengan armonía con la realidad y la cordura evitando la irracionalidad (E) (Navas, 1981). Para los homofóbicos modificar sus esquemas supone un sobre esfuerzo cognitivo que no muchos quieren abordar lo cual dificulta los procesos sociales de ajuste e inclusión de estas poblaciones a nivel local e incluso regional (Andrade et al., 2012). Existen distorsiones notables e ideas mentales fijas que también influyen en la conducta homofóbica, a continuación se enuncian algunas (Tabla 1).

Albert Ellis consideró que las creencias irracionales inducen a extraer conclusiones equívocas disconformes con situaciones específicas, lo cual representa un impedimento para la realización de propósitos propios o ajenos (Almansa, 2011). Desde la perspectiva familiar, la homofobia es un aprendizaje adquirido en sistemas patriarcales que establecen la heterosexualidad como principio básico de las relaciones de familia, debido a la necesidad de pensar la función de procrear como única alternativa de contacto sexual, y distinguir la masculinidad de la femineidad, por lo que la homosexualidad aparece como amenaza ante la continuidad de la familia tradicional (Bustamante, 2009). La homosexualidad es un tema del que se tiene poco conocimiento y que en 
grupos familiares patriarcales suele generar altos niveles de ansiedad, sentimiento de culpa y temor como consecuencia de prejuicios; asimismo estas consecuencias se enmarcan dentro de un plano de expectativas que se tienen de hijos, hermanos, $\mathrm{u}$ otros miembros de la familia (Velásquez, 2008), por ende las actitudes de desprecio y rechazo enseñadas por la familia producen un ambiente de desarrollo hostil que es llevado a otros espacios de interacción por los sujetos, tornándolos violentos e intolerantes ante la identidad sexual que no se ajuste a sus modelos mentales (Valores, 2002). El efecto sobre la persona homosexual es notable en sus emociones, cogniciones, afectos, autoestima y pensamientos irracionales acerca de sí mismo o el mundo (Mott, 2005). Es importante que la familia genere entre sus miembro aprendizajes para integrar la diversidad, al tiempo que las opciones tradicionales de elección de pareja, sin rayar en los extremos y desmitificando la elección homosexual (Bustamante, 2009).

Tabla 1. "Ideas Irracionales acerca de la homofobia". Construido con base en la teoría de Albert Ellis citado por Navas (1981).

\begin{tabular}{|c|c|c|}
\hline Creencia Irracional (Navas, 1981) & Porqué es irracional & Homofobia \\
\hline $\begin{array}{l}\text { No 1: "Es una necesidad extrema } \\
\text { para el ser humano adulto el ser } \\
\text { amado y aprobado por prácticamen- } \\
\text { te cada persona significativa de la } \\
\text { sociedad" }\end{array}$ & $\begin{array}{l}\text { Desear ser aceptados por todos es } \\
\text { una meta imposible, el querer alcan- } \\
\text { zar esta meta es limitarse a vivir la } \\
\text { vida como los demás la desean. }\end{array}$ & $\begin{array}{l}\text { Busca la aprobación, aceptación y } \\
\text { encontrar el amor en los demás, por } \\
\text { ende rechaza a todo aquel que con- } \\
\text { sidera diferente a él. }\end{array}$ \\
\hline $\begin{array}{l}N^{0} 2: \text { "Para considerarse uno mismo } \\
\text { valioso se debe ser muy competen- } \\
\text { te, suficiente y capaz de lograr cual- } \\
\text { quier cosa en todos los aspectos } \\
\text { posibles". }\end{array}$ & $\begin{array}{l}\text { El deseo insaciable por el éxito dis- } \\
\text { trae al individuo de la felicidad que } \\
\text { puede alcanzar, llevándolo a querer } \\
\text { ser superior a los demás. }\end{array}$ & $\begin{array}{l}\text { Miedo al fracaso, a cometer errores, } \\
\text { razón por la cual, no acepta el he- } \\
\text { cho que los seres humanos no son } \\
\text { perfectos. }\end{array}$ \\
\hline $\begin{array}{l}\text { No } 3 \text { : "Cierta clase de gente es vil, } \\
\text { malvada e infame y debe ser seria- } \\
\text { mente culpabilizada y castigada por } \\
\text { su maldad". }\end{array}$ & $\begin{array}{l}\text { Las personas buscan culpabilizar al } \\
\text { otro cuando consideran que comete } \\
\text { errores, se convierten en seres limi- } \\
\text { tados y empiezan a actuar sin tener } \\
\text { conciencia de las consecuencias. }\end{array}$ & $\begin{array}{l}\text { Actúa de manera inadecuada la ma- } \\
\text { yor cantidad de tiempo antes las } \\
\text { personas consideradas fuera de lo } \\
\text { normal. }\end{array}$ \\
\hline $\begin{array}{l}\text { No 4:"Es tremendo y catastrófico el } \\
\text { hecho de que las cosas no vayan por } \\
\text { el camino que a uno le gustaría que } \\
\text { fuesen". }\end{array}$ & $\begin{array}{l}\text { Se centra en todo lo que le desa- } \\
\text { grada o causa malestar en su vida, } \\
\text { rechaza lo diferente y busca grupos } \\
\text { que comportan sus ideales. }\end{array}$ & $\begin{array}{l}\text { No acepta que existen otras incli- } \\
\text { naciones sexuales diferentes a las } \\
\text { "normales". }\end{array}$ \\
\hline $\begin{array}{l}N^{0} 5: " \text { : La desgracia humana se origina } \\
\text { por causas externas y la gente tiene } \\
\text { poca capacidad o ninguna, de con- } \\
\text { trolar sus penas y perturbaciones" }\end{array}$ & $\begin{array}{l}\text { El sentido de las cosas está en la } \\
\text { connotación sentimental que se le } \\
\text { da. Las experiencias influyen en el } \\
\text { pensamiento y crean un malestar } \\
\text { psicológico. }\end{array}$ & $\begin{array}{l}\text { Un homofóbico, es el propio y único } \\
\text { creador de sus sentimientos de re- } \\
\text { chazo que se fortalecen cuando con- } \\
\text { sigue la homogeneidad de grupo. }\end{array}$ \\
\hline $\begin{array}{l}\text { No 6:"Si algo es o puede ser peligro- } \\
\text { so o temible, deberá pensar cons- } \\
\text { tantemente en la posibilidad de que } \\
\text { esto ocurra" }\end{array}$ & $\begin{array}{l}\text { La ansiedad intensa ante la posibili- } \\
\text { dad de que un peligro ocurra, impi- } \\
\text { de afrontar la situación con eficacia } \\
\text { cuando ocurre. }\end{array}$ & $\begin{array}{l}\text { Ansiedad al no poder evitar la diver- } \\
\text { sidad sexual, lo que lo conlleva a } \\
\text { exagerar sus pensamientos y senti- } \\
\text { mientos de rechazo. }\end{array}$ \\
\hline
\end{tabular}


Continuación Tabla 1.

\begin{tabular}{|c|c|c|}
\hline Creencia Irracional (Navas, 1981) & Porqué es irracional & Homofobia \\
\hline $\begin{array}{l}\text { № } 7 \text { :"Es más fácil evitar que afrontar } \\
\text { ciertas responsabilidades y dificulta- } \\
\text { des en la vida" }\end{array}$ & $\begin{array}{l}\text { Evita todo aquello que le genera des- } \\
\text { agrado, aumenta la inseguridad y } \\
\text { desconfianza personal a largo plazo. }\end{array}$ & $\begin{array}{l}\text { Un individuo actúa de manera irra- } \\
\text { cional, se esfuerza en no realizar } \\
\text { actividades, margina y excluye las } \\
\text { personas que considera diferentes. }\end{array}$ \\
\hline $\begin{array}{l}\text { No } 8: " S e \text { debe depender de los de- } \\
\text { más y se necesita a alguien más } \\
\text { fuerte en quien confiar" }\end{array}$ & $\begin{array}{l}\text { Cuanto más se depende de los de- } \\
\text { más, menos se elige por de manera } \\
\text { autónoma. }\end{array}$ & $\begin{array}{l}\text { Depende de su grupo de pares, para } \\
\text { encontrar la aceptación. }\end{array}$ \\
\hline $\begin{array}{l}\text { No 9:"La historia pasada de uno es } \\
\text { un determinante decisivo de la con- } \\
\text { ducta actual". }\end{array}$ & $\begin{array}{l}\text { El pasado, en ocasiones será la } \\
\text { excusa para justiciar los actos de } \\
\text { rechazo, discriminación o rebeldía } \\
\text { hacia los demás. }\end{array}$ & $\begin{array}{l}\text { Las creencias irracionales se crean } \\
\text { en el núcleo familiar, y se reafirman } \\
\text { con el grupo de pares; se ha for- } \\
\text { mado con comportamientos poco } \\
\text { autónomos. }\end{array}$ \\
\hline $\begin{array}{l}\text { No 10:"Uno deberá sentirse muy } \\
\text { preocupado por los problemas y las } \\
\text { perturbaciones de los demás" }\end{array}$ & $\begin{array}{l}\text { Preocupación excesiva por la vida } \\
\text { del otro, sin importar que estas co- } \\
\text { sas afecten o no la propia. }\end{array}$ & $\begin{array}{l}\text { Preocupación por la inclinación se- } \\
\text { xual del otro, no razona ante el daño } \\
\text { que se causa propiamente. }\end{array}$ \\
\hline $\begin{array}{l}N^{0} \text { 11: "Invariablemente existe una } \\
\text { solución correcta para los proble- } \\
\text { mas, y al no encontrarse sobreviene } \\
\text { la catástrofe" }\end{array}$ & $\begin{array}{l}\text { El perfeccionismo conlleva a la reso- } \\
\text { lución de problemas erróneamente, } \\
\text { crea expectativas falsas. }\end{array}$ & $\begin{array}{l}\text { Sesgo moral. Se rige sobre lo que } \\
\text { la sociedad designa como correcto: } \\
\text { relaciones heterosexuales. }\end{array}$ \\
\hline
\end{tabular}

\section{Posición del humanismo respecto a la homofobia}

De acuerdo a Viktor Frankl (1958) lo conveniente es vivenciar y aprender esencialmente todo por sí mismos y, consecutivamente difundir en las personas angustiadas que lo relevante es que la vida tenga expectativas sobre ellos. Por lo tanto, la búsqueda del sentido de la vida por parte de los sujetos homofóbicos, se instaura como una necesidad primaria de supervivencia instintiva, cuyo fin es encontrarse y reconocerse a sí mismo como ser único, logrando dar un significado y valor satisfactorio a sus actos, ya que estará ostentando su propia voluntad (Canal, 2000). Al no encontrarse una aceptación hacia los demás y de sí mismo se puede generar un conflicto en el Yo que muy probablemente desencadenará en una neurosis noógena (angustia espiritual), debida a los conflictos espiritual-éticos, y es así como el sujeto vivenciará un distanciamiento del sentido de la vida y de la existencia (Frankl, 1958).
Aparece entonces un sentimiento de vacío intenso, producto de una crisis existencial y social que el individuo expresa mediante actitudes discriminatorias, sumado el hecho de que el entorno ejercerá una influencia positiva frente a sus pensamientos, reafirmándole todas aquellas creencias inapropiadas, por lo tanto, éste las traducirá en conductas discriminatorias (Moreno, Rodríguez \& Vera, 1996).

Por otra parte, la homofobia como expresión de discriminación se produce y reproduce por los prejuicios que se transmiten en las familias a lo largo del tiempo, estableciendo como punto de referencia de normalidad los estereotipos (Soberon \& Feinzhol, 2007), en otras palabras, las personas están acostumbradas a modelos impuestos por la sociedad misma pero cuando una situación difiere de estos, se le considera amenazante y merecedora de desprecio; en consecuencia una persona homofóbica se adherirá a grupos específicos como la iglesia, clubes de pares, etc. (Espinoza, 2011). 
La homofobia es promovida por la aceptación e inclusión única de quien se somete a los ordenamientos superiores y deja de lado su propio juicio de valor excluyendo y discriminando expresiones desvinculadas de los modelos (Fromm, 1984). A esto se refiere la Etapa preconvencional del Desarrollo moral de Kolbergh, en la que el sujeto juzga los acontecimientos de acuerdo a la recompensa o el castigo que recibe por los demás individuos para de este modo, atribuir la calificación de bueno o malo y en base a ello, actúa con posterioridad para conseguir la aprobación del medio demostrando acatamiento del orden social, etapa denominada por Kolbergh: convencional (Penas, 2008). La relación entre Teoría de Sistemas y psicología define las interacciones familiares como generadoras de estabilidad y cambio, o de inestabilidad y disfuncionalidad personal y social (Andrade, 2015). De modo que una persona para vivir en comunidad de forma ajustada a los contextos y sus variaciones, requiere aprender a fortalecer su capacidad de adaptación, legitimidad, además de generar nuevas vías de comunicación, a fin de modificar la realidad de acuerdo a lo emergente (González, 2000).

\section{DISCUSIÓN}

Durante muchos años la sociedad estuvo regida por un sistema de control político de corte autoritario que modificó las interacciones entre sujetos para generar en ellos acciones que reproduzcan las diversas formas de homogenización y obediencia social, aspecto que se extiende desde el núcleo familiar hasta otras interacciones externas. Entre estas se encuentran las ideas y movimientos machistas-sexistas, que luego tomaron otras formas de representación colectiva. Dichos ejercicios no fueron para siempre ya que el avance en la interpretación de los valores y derechos humanos, ambos motivados por sucesos de violencia de género, hicieron hincapié en la necesidad de resignificación de algunas vivencias y modos de concebir lo sexual y la identidad, como consecuencia emergieron nuevos movimientos individualistas alusivos a la libertad de expresión y de elección sexual (Cárdenas \& Barrientos, 2008). A pesar de ello el mundo continúa presentando expresiones discriminatorias a etnias, culturas, saberes y grupos alternativos, tal es el caso de la discriminación de tendencias sexuales homosexuales (homofobia) sobre las cuales recae el peso de homogenizar la población (Foray, 2002).

Michel Foucault (1974; 1977; 2001) denomina a las tendencias por fuera de la media de normalidad "sexualidades periféricas", porque no solo son externas, sino que responden de subversivamente a formas de subjetivación y dominio de lo sexual. Para la psicología este poder debe tener u referente en el que hacer social, de modo que la existencia de acciones de resistencia demostraría también que existen poderes solidarios, que convocan y reúnen lo diverso en torno a la búsqueda de legitimidad (Rivas 2012), aspecto en el que confluyen las tres escuelas psicológicas estudiadas. Es preciso anotar que Latinoamérica aún presentan manifestaciones homofóbicas pese a los avances en la aceptación de la diversidad sexual, visibles en violencia, agresiones y estigmatizaciones en contra de personas y grupos homosexuales. Para el psicoanálisis la estructuración del superyó en el sujeto homofóbico, deviene de un vínculo familiar que en la infancia generó un registro de exclusión o de rechazo de la sexualidad y el erotismo, es decir una prohibición homosexual que se convirtió a futuro en la voz reguladora/castigadora de la sexualidad, así la ruptura impuesta es a la vez constricción que zanja "castra" el deseo y lo inhibe, volcándolo hacia sí mismo, por lo que una vez ha retornado frustrado se convierte en sentimiento de culpa por la pérdida del objeto, lo que significa también la perdida de sí a razón de la no aceptación de lo homosexual como posibilidad, pues hacerlo significaría revivir la castración originaria (Gómez, 2007). Es notable que a diferencia de los modelos cognitivo y humanista centrados en el aquí y el ahora, la homofobia responde en el psicoanálisis a una historia de exclusión que opera como dispositivo de acción en el sujeto homófobo. 
Las compulsiones agresivas y las prohibiciones son mandatos que devienen de un trasfondo de censura arcaico, que choca en el sujeto con sus deseos de acceder a este tipo de sexualidad, una rotura suturada cuando se enriquece por el peso de exigencias sociales y culturales, lo que desencadena acciones homofóbicas como estrategia de lucha ante la posible desintegración de la identidad masculina. Isidoro Berenstein (1991) afirma que la familia es el núcleo primario de socialización, y en él las experiencias inconscientes ejercen una importante influencia en la movilización de contenidos psíquicos, principalmente de aquellos que modifican las relaciones parentales. Por ello en muchas personas cuya historia de crianza fue problemática (rigidez, castigos, señalamientos, ridiculizaciones, y restricciones), la exclusión de la homosexualidad como dañina o anti-natura genera un registro negativo de la experiencia homosexual, que se decanta en una constricción y dificultad para aceptar relaciones no-heterosexuales como válidas (Badinter, 1993). Para la postura psicodinámica aspectos de la personalidad inconsciente compartidos por identificación con el grupo de pares, pueden propiciar expresiones discriminatorias, a razón "del alma y/o masa colectiva" que cuando se ve cuestionada en sus principios u objetivos, torna impulsiva-agresiva la colectividad (Paniagua \& Fernández, 2007). Efectos de esta movilización de contenidos instintivos puede ser la intolerancia, moralidad arraigada, agresiones, homofobia, descontrol, impulsividad, irritabilidad (Andrade, 2015; Benoist, 2007).

Los homofóbicos crean con su grupo de pares un escenario en el que legitiman sus acciones, dado que en su interior aprenden, se motivan, refuerzan y reproducen ejercicios homofóbicos que se aprueban por el colectivo en tanto intensidad, cronicidad, impulso o permanencia de la agresión. Esta posición es análoga a lo pensado por el modelo cognitivo-conductual, en el que las creencias irracionales parten de inferencias distorsionadas del razonamiento acerca de la sexualidad, en cuyo caso el homofóbico considera de forma rígida que la homosexualidad es en todo y único sentido: contraria a sus principios. Por ello, en defensa de su identidad, acude a racionalizaciones falsas, sobredimensión de la realidad, falacias, visiones catastrofistas de la aceptación de lo homosexual, y evasiones a la integración de personas homosexuales en su existencia. Los homofóbicos suelen ser extremistas y excluyen de su círculo social, a todo aquel que no se amolda a sus ideas rígidas, o que pueden limitar su oportunidad de éxito, dominio y poder social, creando dependencia de su grupo de pares, por el temor a ser rechazo por otros (Almansa, 2011). Asimismo, la homofobia tiene una raíz familiar significativa, especialmente en estilos de crianza patriarcal que suelen alterar el respeto por otros y legitimar la agresión como medio de dominio, respeto y poder (Mott, 2005), a fin de mantener un orden y equilibrio en su dinámica comunicacional interna (Bustamante, 2009).

Estas familias producen un registro rígido/discriminante frente a otros tipos de sexualidad, motivo por el cual aquellos integrantes que no están de acuerdo pueden experimentar un desajuste funcional a razón de conflictos derivados del choque entre sus creencias personales, y la ley familiar heterosexual irrevocable (Almansa, 2011), tal es el caso de personas homosexuales que temen hablar de su condición a la familia a razón de prejuicios, señalamientos, e intimidaciones, además del desconocimiento, por lo que sufren de forma frecuente discriminaciones al interior de su hogar (Rodríguez, 2012), así como también en su ambiente comunitario y social, lo cual tiene efectos permanentes en la salud mental y física de estas personas (Herek \& Berrill, 1992). La psicología humanista señala la existencia de un sentido de vida alterado en el sujeto homofóbico, dicho sentido configura el modo en que cada ser vive percibe y experimenta la realidad de su vida, la cual, en los homofóbicos se orienta hacia la búsqueda constante de placer y evitación del dolor, además de la individualidad e ilegitimidad de aquello que considera contrario a sí mismo, $\mathrm{y}$ vive sin percatarse de la autodestrucción que 
propende, cuando niega la legitimidad del otro en la convivencia (Maturana, 1990). Para Viktor Frankl (1958) el sentido de vida se hace presente en cuanto el sujeto es capaz de legitimarse, de amarse, de reconocerse a sí mismo como único y no en la pretensión de reafirmarse en el otro o en la exorbitante necesidad de afiliación.

De este modo la neurosis noógena o neurosis procedente de la pérdida del sentido de la vida, también explica la homofobia la cual es en todo sentido un sufrimiento a causa de vivir el sinsentido. Desde el lugar de la exclusión del otro, la percepción errónea de la realidad genera en la persona homofóbica una sensación de vacío y de vaciado, en las que emergen sentimientos de culpa, ira, impulsividad, desolación e inestabilidad emocional tanto hacia otros como hacia sí mismo (Moreno, Rodríguez \& Vera, 1996). Para Viktor Frankl la negación del otro no solo aliena sus posibilidades como sujeto, sino su humanidad misma, lo cual es también la negación de sí porque cada persona puede reconocerse como tal en la analogía y la diferencia; en este sentido toda acción de rechazo, exclusión, limitación y anulación derivada de la homofobia, implica a su vez la anulación de la legitimidad del otro (Hamilton, 2007). Los enfoques psicológicos consideran la homofobia una conducta de carácter inconsciente en sus motivaciones, y como efecto del aprendizaje y reforzamiento social realizado por diversos grupos con los que se interactúa o como una elección que cuando se frustra o permanece en anonimato, genera neurosis noógena, es decir una pérdida del sentido vital, además de la vivencia de ilegitimidad e inautenticidad. De acuerdo a lo propuesto ninguna postura es mejor que la otra en términos explicativos, sin embargo es claro que el psicoanálisis y el conductismo tienen un enfoque causalista, pero su diferencia radica en el tiempo de emergencia más que en la interacción entre factores precipitantes.

Así, mientras el modelo psicoanalítico explica la homofobia desde motivaciones inconscientes, frustraciones, complejos invertidos
(Edipo-Electra), fallas en la estructura narcisista (Babiszenko \& Lutereau, 2011). Para el modelo conductista, la homofobia se aprende y se refuerza en el encuentro social con agentes y personas discriminantes que ejercen un poder especial sovre el sujeto, haciendole pertenecer a un grupo en el que cumple sus deseos de aprobación, genera ansiedad por el control, y obtiene reconocimiento de la reproducción de las acciones homófobas (Almansa, 2011; Rivas, 2012; Navas, 1981). Correlativamente, en el humanismo, el reconocimiento existencial es trascendental, así las personas que no reconocen su homosexualidad tienden a sufrir crisis existenciales, del mismo modo que aquellos que no reconocen empáticamente el daño ocasionado a otros, viven una crisis permanente de identidad social. La autenticidad del agresor homofóbico no se encuentra en reconocer su homofobia, sino en el hecho de comprender que sus acciones van en detrimento de la humanidad y en ese sentido de su Self (sí mismo), las conductas homofóbicas no sirven a la voluntad de la humanidad, sino a la voluntad del amor, el cual se reduce a la exclusión de todo aquello que es considerado distinto y que atenta contra la rigidez de los pensamientos y orientaciones consideradas como válidas e inmutables.

\section{CONCLUSIONES}

Las personas homofóbicas logran reponerse al interiorizar la exclusión como mandato inconsciente, distorsión cognitiva o como estilo de vida, terminan tolerando la injusticia de la discriminación, y por tanto reproducen la exclusión como estrategia de supervivencia y de legitimidad de sus acciones, las cuales se encuentran claramente orientadas a la eliminación del otro. La discriminación emerge de forma estructural y estructurante en la existencia y motivaciones de personas y grupos, con base en la concurrencia de juicios de valor y experiencias de exclusión, que se gatillan en presencia de un grupo que refuerza estas conductas. Aspectos como la identidad y la identificación a nivel psicológico fundamentan tanto la posición de las personas homosexua- 
les, como la de los homófobos, la discrepancia en ambos grupos radica en la imposibilidad de encontrar puntos comunes de diálogo y acuerdo, ya que es precisamente esta carencia lo que desata las acciones de homofobia y de inaceptación de la identidad del otro. La homofobia configura imaginarios sociales a través de las múltiples representaciones extremas de vivir los géneros, la masculinidad, la feminidad y la identidad. No solo se reduce a las prácticas de fuerza y agresiones teledirigidas, ya que conlleva un enorme potencial simbólico, real e imaginario que resulta determinante en las acciones de repetición y conciencia de legitimidad de las agresiones y de la violencia en contra de personas homosexuales.

Para los modelos psicológicos, su génesis no se describe en cuanto patología, ya que es la conformación social de esta conducta lo que prima en su ejercicio; así, entre más identidad, reforzamiento o elección para ejecutarla exista, mayor es la disposición emocional que tiene la persona homófoba para agredir a otros que considera su alter-ego, es decir, la negación de sí. Los modelos psicológicos entienden la homofobia a partir de sus disposiciones teóricas, sin embargo caen en reduccionismo notables en términos de etiogénesis; correlativamente el enfoque humanista se aproxima al sentir de ambos actores (agresoragredido) denotando la pérdida del sentido vital como ejercicio en el homofóbico y como efecto en la mayoría de las víctimas. Se requiere en torno a dicho vacío una visión relación bajo el marco transdisciplinario, que reúna conceptos, experiencias, lógicas, ideas, representaciones, etc., que ponga a dialogar las disciplinas y permita una visión nos sesgada del fenómeno.

El ejercicio de la homofobia implica la activación y uso de representaciones sociales ligadas a la discriminación aprendida, asumida y ejercida, pasando por un intento de subversión de identidades de género alternativas, hasta la anulación total del otro, por lo que la aceptación y legitimidad quedan excluidos del discurso de la persona homófoba. Asimismo existe en toda discriminación una exaltación del biopoder, es decir del po- der sobre la regulación de la vida, que se extiende hasta el cuerpo y la subjetividad, y que en gran medida manipula la condición de género asociándola a lo natural en cuanto relación heterosexual, mientras la homosexualidad sería patológico o contra-natura. La homofobia es una de las formas de xenofobia política de minorías sociales históricamente excluidas de los beneficios del estado social democrático y de derecho, y responde a nuevas formas de opresión social, coacción y linealización de las interacciones tradicionales.

Las conductas de discriminación pueden ser tomadas por muchos como acciones para conseguir un mayor poder social y reconocimiento político y social, mientras para otros puede responder a un legado que ha sido heredado y que acompaña al xenófobo como estigma, por tanto una forma de librarse de dicha condición es suprimiendo al otro, por lo que toda exclusión es una defensa, una respuesta ante la posibilidad de ser excluido, discriminado y censurado. La homofobia remite a un temor ante algo que se niega, aspecto en el que confluyen los tres modelos psicológicos aquí enunciados. Aunque se trate de aprendizajes, problemas arcaicos/motivaciones inconscientes, o pérdida del sentido vital, la homofobia tiene en estos enfoques consecuencias análogas: la anulación permanente del otro, la evidencia de un persistente proceso de ilegitimidad y la persistencia de secuelas biopsicosociales que afectan de forma constante su calidad, expectativa y estilo de vida de quienes son víctimas de este flagelo.

\section{REFERENCIAS BIBLIOGRÁFICAS}

Almansa, C. (2011). Las doce creencias irracionales que sustentan la neurosis (A. Ellis) y los doce curadores (E. Bach): estudio de su correspondencia. congreso sedibac de Terapia Floral, 1-36.

Andrade, J. A., Quintero, L. \& Téllez, C. (2012). Aproximaciones explicativas de la homosexualidad desde tres perspectivas psicológicas. Psicopedia hoy, 14 (5). Recuperado de http://psicopediahoy.com/ homosexualidad-perspectivas-psicologicas/ 
Andrade, J. A. (2011). La intervención grupal: una lectura de los conceptos de enrique pichón riviere: artículo de reflexión investigativa. Revista Electrónica de Psicología Iztacala, 14(3), 194-228.

Andrade, J. A. (2015). Introducción a la psicología social. Psicología social y conflicto. Apuntes para la construcción de una psicología social compleja bajo un dispositivo clínico-social. Colombia: FUNDES.

Araya, S. (2002). Las representaciones sociales: ejes teóricos para su discusión. Costa Rica: Facultad latinoamericana de ciencias sociales Ardila, R. (1998). Homosexualidad y Psicología. Bogotá: El manual moderno.

Asociación Internacional de Lesbianas, Gays, Bisexuales, Trans e Intersexuales -ILGA (2013). Recuperado de http://old.ilga.org/ Statehomophobia/ILGA_Homofobia_de_ Estado 2013.pdf

Babiszenko, D. \& Lutereau, L. (2011). La homosexualidad femenina en el psicoanálisis de J. Mc Dougall. Recuperado de http://www.apdeba.org/wp-content/uploads/Babiszenko.pdf

Badinter, E. (1993). XY, la identidad masculina. Bogotá: Norma.

Ballero, M. (2008). Informe anual sobre derechos humanos de personas trans, lesbianas, gays y bisexuales en el Perú 2008. Perú: Red Perana de TLGB, Promsex.

Benoist, A. D. (2007). Gustav Le Bon. En Psicología de las masas por Gustav Le Bon. Recuperado de http://disenso.info/wp-content/ uploads/2013/06/Psicologia-de-las-masas-G.Le-Bon.pdf

Berenstein, I. (1991). Reconsideración del concepto de vínculo. Los vínculos. Revista de Psicoanálisis, AP de Buenos Aires, 13(2), 219-235.

Bustamante, A. (2009). "Homoerotismo y homofobia en Colombia: una visión histórica». En: III Seminario Internacional sobre Familia, Universidad de Caldas, abril de 2008. Recuperado de http://www.ucaldas.edu.co/ docs/seminario familia/HOMOEROTISMO HOMOFOBIA_COLOMBIA_Walter_ Bustamante.pdf

Canal, J. Y. (2000). El sentido de las propuestas existenciales. Universidad nacional de Colombia. Recuperado de http://www.docentes.unal.edu.co/jyanezc/docs/la\%20busqueda $\% 20$ desentido $\% 20$ en $\% 201 \mathrm{a} \% 20$ obra $\% 20$ de\%20viktor\%20frankl.pdf

Cárdenas, M. \& Barrientos, J. (2008). Actitudes explícitas e implícitas hacia los hombres homosexuales en una muestra de estudiantes universitarios en Chile. Psykhe 17(2), 17-25.

Carrillo, R. A. (2006). Diccionario de la sexualidad, tomo 1. Mexico: Editorial del Valle.

Centro Para la Educación y Prevención del SIDA -CEPRESI (2013). Encuesta de Opinión sobre Homofobia dirigida a Estudiantes Universitarios en Cinco Departamentos de Nicaragua. Recuperado de http://www.cepresi. org.ni/files/doc/1246385516_Resultados $\% 20$ de $\% 20$ Encunesta $\% 20 \mathrm{de} \% 20$ Opinion $\% 20$ sobre $\% 20$ Homofobia $\% 20$ dirigida $\% 20 a \% 20$ Estudiantes.pdf

Chamberland, L. \& Lebreton, C. (2012). Réflexions autour de la notiond'homophobie: Succèspolitique, malaisesconceptuels et applicationempirique. Reflexiones sobre la noción de homofobia: éxito político, malestares conceptuales y aplicaciones empíricas. Nouvelles Questions Féministes.

Christopher, F. S. \& Sprecher, S. (2000). Sexuality in marriage, dating, and other relationships: A decade review. Journal of Marriage and Family, 62(4), 999-1017

Colombia diversa -CD (2013). Cuando la guerra se va, la vida toma su lugar. Recuperado de, http://colombiadiversa.org/colombiadiversa/documentos/informes-dh/colombia-diversainforme-dh-2013-2014-resumen.pdf

Espejo, J. C. (2009). Equívocos del lenguaje: homoerotismo en lugar de homosexualidad. Revista ALPHA, (29), 143-154. 
Espejo, J. C. (2012). Componentes ideológicos de la homofobia. Red de Revistas Científicas de América Latina, el Caribe, España y Portugal, 7(26), 85-106

Espinoza, R. (2011). Abordaje actual de la homosexualidad en una visión personalista *. Anales de la ciencia medica, I Cátedra de Clínica Quirúrgica, Hospital de Clínicas, Facultad de Ciencias Médicas - UNA, 42(2), 45-59.

Foray, D. (2002). La sociedad del conocimiento. UNESCO, Revista internacional de ciencias sociales, 172, 1-33. Recuperado de http://www. unesco.org/new/fileadmin/MULTIMEDIA/ HQ/SHS/pdf/171-fulltext171spa.pdf

Foucault, M. (1974). Sexualité et politique, en Michel Foucault. Ditsetécrits I, 1954-1975, Paris, Gallimard.

Foucault, M. (1977). No al sexo rey. Entrevista por Bernard Henry-Levy, en Michel Foucault: Un diálogo sobre el poder y otras conversaciones. Madrid: Alianza Editorial.

Foucault, M. (2001). Sexualität und Wahrheit, en Michel Foucault. Ditsetécrits II, 1976-1988, Paris: Gallimard.

Frankl, V. (1958). El hombre en busca de sentido. Barcelona: Editorial Herder.

Freud, S. (1923). El Yo y el ello. Buenos Aires: Amorrortu Editores.

Freud, S. (1978). Lo Inconsciente. Buenos Aires: Amorrortu Editores.

Fromm, E. (1984). Sobre la desobediencia y otros ensayos. Buenos Aires: Editorial Paidós, SAICF.

Gaione, C. (2009). Pluralidad de los orígenes del superyó... ¿identificaciones en conflicto? Revista Uruguaya de Psicoanálisis, 109, 125 148, recuperado de http://www.apuruguay.org/ apurevista/2000/16887247200910909.pdf

García, C., Quiroga, X., Morgan, A. Paller. \& Marrero, D. (2011). Transtextualizando la Diversidad Sexual. Estocolmo: Publicaciones LatiCe.

Gómez, M. M. (2007). Violencia, homofobia y psicoanálisis: entre lo secreto y lo público. Revista de Estudios Sociales, 28(1), 72-85

González, I. (2000). Las crisis familiares. Revista Cubana de Medicina General
Integral, 16(3), 280-286. Recuperado de http://scielo.sld.cu/scielo.php?pid=S086421252000000300010\&script $=$ sci_arttext

Hamilton, L. (2007). Trading on heterosexuality: Collegue women's gender strategies and homophobia. Gender Society, 21(2), 145-172.

Herek, G. \& Berrill, K. (Eds). (1992). Hate Crimes: Confronting Violence Against Lesbians and Gay Men. Newbury Park, California: SagePublications.

Instituto Nacional contra la Discriminación, la Xenofobia y el Racismo -INADI (2015). Recuperado de http://inadi.gob.ar/institucional/ Izaguirre, I. (2008). Violencia Social y Derechos Humanos. Buenos Aires, Argentina: Eudeba Editores.

Lacarde, M. (1996). Identidad de género y derechos humanos. La construcción de las humanas. En Estudios Básicos de Derechos Humanos. Instituto Interamericano de Derechos Humanos: IIDH, San José, Costa Rica. 86-12

Lamas, M. (2000). Diferencias de sexo, género y diferencia sexual. Revista Cuicuilco, 7(18), 1-24.

Maturana, H. (1990). Emociones y lenguaje en educación y política. 2da edición. Chile. Editorial universitaria.

Mondragón, J. M. (2009). Intolerancia a la diversidad sexual y crímenes por homofobia Un análisis sociológico. Sociologica, 24(69), 123-156.

Moreno, G., Rodríguez, J. \& Vera, J. (1996). La participación laboral femenina y la discriminación salarial en España (1990-1991). España: Consejo Económico y Social.

Moscovici, S. (1999). Psicología social: pensamiento y vida social; psicología social y problemas sociales. Editorial Paidós: Barcelona.

Mott, L. (2005). Las raíces de la homofobia en américa latina, en "Homofobia en America Latina historia Adolescente Gay". 1-6. Recuperado de http://www.censida.salud.gob. $\mathrm{mx} /$ descargas/drhumanos/luizmott.pdf

Navas, R. (1981). Terapia racional emotiva. Revista Latinoamericana de Psicología. 13(1), 75-83. Recuperado de http://www.redalyc.org/ pdf/805/80513105.pdf 
Organización de las Naciones Unidas para la Educación, la Ciencia y la Cultura UNESCO (2015). El acoso homofóbico, (en línea). Recuperado de http://www.unesco. org/new/es/education/themes/leading-theinternational-agenda/health-education/ homophobic-bullying/

Organización de las naciones unidas para la educación, la ciencia y la cultura - UNESCO (2015a). Respuestas del Sector de Educación Frente al Bullying Homofóbico. Cuadernillo 8. Recuperado de http://unesdoc.unesco.org/ images/0022/002229/222918S.pdf

Organización Panamericana de la Salud OPS (2006). Campañas contra la homofobia en Argentina, Brasil, Colombia y México. Recuperado de http://www.paho.org/hq/index.php?option $=$ com_docman $\&$ task $=$ doc view\&gid $=23850 \&$ Itemid

Ortega, P. (2011). Psicoanálisis, la familia y la educación. La Nueva Escuela Lacaniana del campo Freudiano. México D.F. Recuperado de: http://www.nel-mexico.org/articulos/seccion/varite/edicion/ Ninos-y-adolescentes-del-nuevo-siglo/408/ El-psicoanalisis-la-familia-y-la-educacion

Paniagua, C. \& Fernández, J. (2007). Psicología de las masas y violencia. Ars Médica. Revista de Humanidades, 2, 235-264. Recuperado de http://www.dendramedica.es/revista/v6n2/ Psicologia_de_las_masas_y_violencia.pdf

Paulón, E. (2010). Matrimonio para todas y todos Ley de igualdad. Argentina: Federación Argentina LGBT.
Penas, S. (2008). Aproximación a los valores y estilos de vida de los jóvenes de 13 y 14 años de la provincia de a coruña. Tesis doctoral, Facultad de psicología. Universidad de Santiago de Compostela. Recuperado de https://dspace.usc. es/bitstream/10347/2465/1/9788498870879 content.pdf

Rodríguez, T. (2012). Sobre Homofobia y/o ser enfermero. Revista de Ciencias Médicas Scielo.16(1), 2-7

Rivas, C. Z. (2012). Psicología, sociedad y equidad: Aportes y desafios. Santiago de Chile: Salesianos Impresores S.A.

Rodríguez-Castro, Y., Lameiras-Fernández, M., Carrera-Fernández, V. \& Vallejo-Medina, P. (2013). Validación de la Escala de Homofobia Moderna en unamuestradeadolescentes. Revista Anal. Psicol., 29(2), 523-533. Recuperado de http://scielo.isciii.es/scielo.php?pid=S021297282013000200024\&script $=$ sci_arttext

Soberon, G. \& Feinzhol, D. (2007). Homofobia y salud. Mexico: Secretaria de salud comisión nacional de bioetica. México: Memorias CNB. Valores, E. E. (2002). El respeto a la diferencia por orientación sexual. España: Xente Gai Astur - "Xega" - Xega Xoven.

Velásquez, A. (2008). VI Informe Anual: Derechos Humanos Minorías Sexuales Chilenas. Chile: editorial Movilh. 